OPEN ACCESS

Edited by:

Atsushi Otsuka,

Kyoto University, Japan

Reviewed by:

Daniel Olive,

Aix Marseille Université, France

Yasuhiro Nakamura,

Saitama Medical University International Medical Center,

Japan

*Correspondence:

Phil F. Cheng

phil.cheng@usz.ch

Specialty section:

This article was submitted to

Cancer Immunity and Immunotherapy,

a section of the journal

Frontiers in Oncology

Received: 02 March 2018

Accepted: 08 May 2018

Published: 29 May 2018

Citation:

Hogan SA, Levesque MP and

Cheng PF (2018) Melanoma

Immunotherapy:

Next-Generation Biomarkers.

Front. Oncol. 8:178.

doi: 10.3389/fonc.2018.00178

\section{Melanoma Immunotherapy: Next-Generation Biomarkers}

\author{
Sabrina A. Hogan ${ }^{1,2}$, Mitchell P. Levesque ${ }^{1,2}$ and Phil F. Cheng ${ }^{1,2 *}$ \\ ${ }^{1}$ Department of Dermatology, UniversitätsSpital Zürich, Gloriastrasse, Zurich, Switzerland, ${ }^{2}$ Faculty of Medicine, \\ Universität Zürich, Zürich, Switzerland
}

The recent emergence of cancer immunotherapies initiated a significant shift in the clinical management of metastatic melanoma. Prior to 2011, melanoma patients only had palliative treatment solutions which offered little to no survival benefit. In 2018, with immunotherapy, melanoma patients can now contemplate durable or even complete remission. Treatment with novel immune checkpoint inhibitors, anti-cytotoxic T-lymphocyte protein 4 and anti-programmed cell death protein 1, clearly result in superior median and longterm survivals compared to standard chemotherapy; however, more than half of the patients do not respond to immune checkpoint blockade. Currently, clinicians do not have any effective way to stratify melanoma patients for immunotherapies. Research is now focusing on identifying biomarkers which could predict a patient's response prior treatment initiation (or very early during treatment course), in order to maximize therapeutic efficacy, avoid unnecessary costs, and undesirable heavy side effects for the patient. Given the rapid developments in this field and the translational potential for some of the biomarkers, we will summarize the current state of biomarker research for immunotherapy in melanoma, with an emphasis on omics technologies such as next-generation sequencing and mass cytometry (CyTOF).

Keywords: melanoma, immunotherapy, biomarkers, next-generation sequencing, review literature as topic

Immunotherapy has revolutionized the management of metastatic melanoma. Prior to 2011, the median survival for metastatic melanoma was 9 months, compared to greater than 18 months in 2017 (1). Patients now benefit from novel immune checkpoint inhibitors (ICIs), anti-cytotoxic T-lymphocyte protein 4 (CTLA-4) and anti-programmed cell death protein 1 (PD-1). From the latest survival data of the Checkmate 067 trial, progression-free survival (PFS) for ipilimumab is 2.9 months, for nivolumab 6.9 months, and for the combination of nivolumab and ipilimumab 11.5 months. Overall survival (OS) of the ipilimumab group was 19.9 and 37.6 months for the nivolumab group. Median OS was not reached in the combination nivolumab and ipilimumab group with a minimum follow-up time of 36 months (2-6). Although OS is extended, not all patients benefit from immunotherapy. Response rates for ipilimumab range from $11 \%$ to $19 \%(4,5)$ and for pembrolizumab or nivolumab from $33 \%$ to $44 \%(2,6,7)$. These new ICIs clearly show superior median and long-term survivals compared to standard chemotherapy; however, more than half of the patients do not respond to immune checkpoint blockade. Currently, there are no clinically approved biomarkers to aid in patient selection in melanoma. In this review, we seek to delineate the current state of biomarker research for immunotherapy in melanoma, with an emphasis on omics technologies such as next-generation sequencing (NGS) and mass cytometry (CyTOF). Given the urgent clinical need for such biomarkers, we decided to focus on human studies only, which we think are more clinically relevant. 


\section{IMMUNE CHECKPOINTS}

CTLA-4 and PD-1 are two immune checkpoints regulating immune homeostasis. CTLA-4 is a negative regulator of T-cell priming that acts to control naïve T-cell activation by competing with the co-stimulatory molecule CD28 for binding to shared ligands CD80 and CD86 on antigen-presenting cells (APCs) in the lymph node (8). Ipilimumab, a monoclonal antibody against CTLA-4, was the first agent approved for the treatment of unresectable or metastatic melanoma that showed an OS benefit in a randomized phase III trial (4). PD- 1 is a T-cell exhaustion marker which is upregulated by $\mathrm{T}$-cells upon activation during priming or expansion and binds to one of two ligands: programmed cell death 1-ligand 1 (PD-L1) and -ligand 2 (PD-L2) (9-11). Pembrolizumab and nivolumab are monoclonal antibodies against PD-1 that have both shown OS benefit in randomized phase III trials and are approved for the treatment of metastatic melanoma $(2,7)$. Furthermore, nivolumab and pembrolizumab have both improved OS compared with ipilimumab in metastatic melanoma patients that are naïve to both agents. Combination therapy with ipilimumab and nivolumab has demonstrated additional clinical activity with objective response rates ranging from $50 \%$ to $60 \%$ and improved OS compared to ipilimumab alone. Although ipilimumab, nivolumab, and pembrolizumab have significantly improved the survival of melanoma patients, there are major toxicities associated with the use of these drugs [reviewed in Ref. (12)]. Grade 3 and higher adverse events are seen in about $20 \%$ of patients treated with ipilimumab, in $15 \%$ of patients treated with nivolumab, and in $50 \%$ of patients treated with the combination of both drugs (6). As these therapies result in objective responses for only a subset of patients, there is a crucial need to identify biomarkers that can potentially predict the efficacy of anti-CTLA-4 or anti-PD-1 treatment or identify a specific subset of patients who may benefit from immunotherapy. A summary of current potential biomarkers for immunotherapies in metastatic melanoma patients is listed in Figure 1.

\section{CLINICAL BIOMARKERS}

Approved markers for melanoma monitoring have not substantially evolved over the past decade. Clinicians have mainly used the TNM staging system as a diagnostic and prognostic indicator. In 2009, lactate dehydrogenase (LDH) was shown to be an independent predictor of survival in melanoma and was therefore added to the AJCC guidelines (13). Accelerated metabolism in cancer cells requires increased glycolysis that creates a high amount of LDH as a byproduct, which is therefore a robust proxy to assess tumor burden (14). It is the only accepted serum biomarker with prognostic value for OS in melanoma (15). In the context of immunotherapies, elevated $\mathrm{LDH}$ is a negative prognostic marker for patients treated with ipilimumab (16) and with pembrolizumab $(17,18)$. However, subgroup analysis of anti-PD- 1 treated cohorts recently pointed out that LDH level is not correlated with the duration of response (KEYNOTE-006). Indeed, once patients show response to the treatment, the $\mathrm{LDH}$ level is not associated with the duration of the remission period.
As described by Diem et al. in a study specifically assessing the role of LDH as a marker for anti-PD-1 therapy, $\mathrm{LDH}$ is nevertheless a useful marker to monitor disease progression and help treatment decisions (19).

Another well-known marker to monitor melanoma is S100, which is a good indicator of advanced clinical disease stage (20). S100 was shown to be predictive of response to anti-CTLA-4 (16). However, similar to LDH, S100 seems to mainly be a proxy of disease stage, able to highlight very ill patients who are more unlikely to respond to the treatment due to the high tumor burden of the disease, but not actually able to predict response to immunotherapies. The same is true for the number of organs involved, which was another potential marker, proposed by Diem et al., to stratify patients prior to anti-CTLA-4 therapy (21).

C-reactive protein (CRP) was described as a negative prognostic factor for anti-CTLA-4 treatment (22). Unlike LDH and $\mathrm{S} 100, \mathrm{CRP}$ is directly related to immune response. However, it is a general marker of inflammation and is not specific to melanoma, ergo, an increase in CRP levels may also be the result of any other ongoing infection (23). For anti-PD-1 therapy, intra-tumoral PD-L1 expression, evaluated by immunohistochemistry, has been assessed as a predictive biomarker. The results have been inconclusive due to a lack of standards for PD-L1 "positivity". Different antibodies and different evaluation criteria have been used for PD-L1 expression in clinical trials. Some studies have used a $>5 \%$ cutoff (Checkmate-066 and Checkmate-067), whereas others have used $>1 \%$ cutoff (KEYNOTE-006). In the Checkmate-066 trial, both PD-L1 negative and positive patients had better outcomes than chemotherapy-treated patients, suggesting that PD-L1 status was not a relevant stratification marker (2). More research will be needed to standardize the assessment of PD-L1 expression for it to become a biomarker for anti-PD-1 therapy in melanoma. Blood markers which hold the most potential toward predicting response to immunotherapies are immune cell populations. Indeed, they are either themselves part of or directly influencing the immune response against the tumor. The different findings related to blood cytology as a biomarker for immunotherapy in melanoma are summarized in Figure 1. Briefly, for anti-CTLA-4 treatment, absolute neutrophil count, absolute lymphocyte count, neutrophil to lymphocyte ratio, absolute eosinophil count, relative lymphocyte count (RLC), absolute monocyte count, antibodies against NY-ESO1, T-regulatory cell count, and myeloid-derived suppressor cell (MDSC) count have been described as predictive biomarkers. In anti-PD-1-treated patients, RLC, relative eosinophil count (REC), and MDSC count seem to hold some predictive potential prior to treatment initiation. In addition, increased serum levels of TGF $\beta$ and increased frequency of Th9 cells in the peripheral blood were detected in responders to nivolumab prior to therapy initiation (24). Unfortunately, most of the studies were performed on small cohorts and the results have not been verified in larger prospective trials (25). Although guidelines have been published about how to best perform biomarker studies (26), most research groups have different evaluation criteria. In this review, we sought to document the most relevant biomarkers associated with immunotherapy outcome in melanoma patients. For a systematic review of clinical biomarkers, see Ref. (18). 


\begin{tabular}{|c|c|c|c|c|c|c|c|}
\hline & Drug & \# patients & Markers & Correlation & Outcome & Method / Threshold & Reference \\
\hline \multirow{10}{*}{ CyTOF } & \multirow{2}{*}{ Ant-PD1 } & \multirow{2}{*}{4} & $\uparrow \mathrm{CD}^{+}$and $\mathrm{CD} 4^{+} \mathrm{CD} 8^{+}$T-cells atter $6-13$ weeks oftreatment & \multirow{2}{*}{+} & \multirow{2}{*}{ Response } & \multirow{2}{*}{$\begin{array}{l}\text { High dimentional clustering } \\
\text { viSNE and SPADE }\end{array}$} & \multirow{2}{*}{ Takeuchi et al 2017} \\
\hline & & & $\dagger$ Central Memory T-cell after 6-13 weeks of treatment & & & & \\
\hline & Ant-PD1 & \multirow[t]{2}{*}{20} & $1 \mathrm{CD}^{*}$ and $\mathrm{CDB}^{*} \mathrm{~T}$-cells & \multirow[t]{2}{*}{+} & Response & High dimentional clustering & Krieg et al. 2018 \\
\hline & Ant-PD1 & & $\begin{array}{l}\text { TClassical monocyes frequency } \\
\text { TCD8 expressig LL-2 }\end{array}$ & & Response & $\begin{array}{c}\text { Flowsom } \\
\text { High dimentonal clustering }\end{array}$ & Hiniker etal. 2016 \\
\hline & $\begin{array}{l}\text { Ant-CTLA4 + } \\
\text { Ant-PD1 }\end{array}$ & 9 & I Transitional Memory T-cells frequency & + & Response & $\begin{array}{c}\text { SPADE } \\
\text { DVS Cylobank software }\end{array}$ & Das et al. 2015 \\
\hline & \multirow{5}{*}{ Various } & \multirow{5}{*}{28} & $\lceil$ APC-like population frequency & + & \multirow{5}{*}{ Overall survival } & \multirow{5}{*}{ Matrix boolean analysis } & \multirow{5}{*}{ Wistuba-Hamprecht et al. 2017} \\
\hline & & & $\uparrow$ MDSC-like population frequency & - & & & \\
\hline & & & Even abundance of MDSC- and APC-like cells & + & & & \\
\hline & & & I Early differentiated CD4 correlate with & - & & & \\
\hline & & & T Highly cyototoxic NK cell population & + & & & \\
\hline \multirow{3}{*}{$\begin{array}{l}\text { TCR } \\
\text { profiling }\end{array}$} & Ant-PD1 & 12 & I TCR richness and evenness & - & Response & Multiplex PCR - CDR3 TCR $\beta-V J$ & Postow et al. 2015 \\
\hline & Ant-CTLA4 & 21 & $\begin{array}{l}\text { T Frequency of clonotypes maintained overtime } \\
\text { I Decrease in the number of clones overtime }\end{array}$ & * & Overall survival & NGS - CDR3 TCRß-VDJ & Cha et al. 2014 \\
\hline & Ant-CTLA4 & 21 & $\begin{array}{l}\text { T TCR repertoire diversity after } 30-60 d \text { of treatment } \\
T C R \text { repertoire richness and diversity afer } 30-60 d \text { of treatment }\end{array}$ & + & $\begin{array}{c}\text { Response } \\
\text { Toxicity }\end{array}$ & NGS - CDR3 TCR $\beta-V$ & Robert et al. 2014 \\
\hline NGS & Anti-CTLA4 & 360 & $\begin{array}{l}15 \text { gene classifier (ADAM17, CDK2, CDKN2A, DPP4, ERBB2, HLA- } \\
\text { DRA, ICOS, ITGA4, LARGE, MYC, NAB2, NRAS, RHOC, TGFBI. } \\
\text { TIMP1 }\end{array}$ & + & $\begin{array}{l}\text { Overall suruval } \\
\text { and response }\end{array}$ & QPCR & Friedlander et al 2017 \\
\hline & Anti-PD1 & 512 & I Lactate dehydrogenase (LDH) & + & Overall survival & LDH ratio $>2.5$ & $\begin{array}{l}\text { Weide etal. } 2016 \\
\text { Diem et al. } 2016\end{array}$ \\
\hline & Ant-PD1 & 88 & † Myeloid derived supressor cells (MDSC) & $\cdot$ & $\begin{array}{c}\text { Overall survival } \\
\text { PFS }\end{array}$ & $12.60 \%$ & Weber et al. 2016 \\
\hline & Ant-PD1 & 512 & T Relative Iym phocye counts $(\mathrm{RLC})$ & + & Overall sunvival & $17.50 \%$ & Weide etal. 2016 \\
\hline & Ant-PD1 & 512 & i Relative eosinophils counts (REC) & + & Overall survival & $1.50 \%$ & Weide etal. 2016 \\
\hline & Ant-PD1 & 46 & $\uparrow$ TGF $\beta$ in serum & + & Response & & Nonomura et al. 2016 \\
\hline & Ant-PD1 & 46 & T Th9 earlyduring treatment & + & Response & & Nonom ura et al. 2016 \\
\hline & & 311 & I Lactate dehydrogenase (LDH) & & Response & $2 \times U L N$ & Wolchok et al. 2016 \\
\hline & Ant-CTLA4 & 200 & I Lactate dehydrogenase (LDH) & + & Overall sunival & ULN & Martens et al. 2016 \\
\hline & & 134 & I Lactate dehydrogenase (LDH) during treatment & & Overall survival & Continuous variable & Diem et al. 2015 \\
\hline & Ant-CTLA4 & $\begin{array}{ll}100 \\
95\end{array}$ & $\begin{array}{l}\text { I absolute Iymphocye count (ALC) } \\
\text { i absolute Iym phocyte count (ALC) during treatment }\end{array}$ & + & $\begin{array}{c}\text { PFS } \\
\text { Response } \\
\end{array}$ & $1 \times 10^{9}$ & $\begin{array}{l}\text { Alexander et al. } 2014 \\
\text { Simeone et al. } 2014\end{array}$ \\
\hline Serum, & Ant-CTLA4 & 720 & † Absolute neutrophil count (ANC) & - & $\begin{array}{l}\text { Overall sunvival } \\
\text { PFS }\end{array}$ & 7500 & Ferrucci et al. 2016 \\
\hline Blood & Ant-CTLA4 & 200 & T Absolute eos inophil count (AEC) & + & Overall sunvival & 50/ug & $\begin{array}{l}\text { Martens et al. } 2016 \\
\text { Weide etal. } 2016\end{array}$ \\
\hline markers & Anti-CTLA4 & 200 & $\uparrow$ Relative Iym phocyte counts (RLC) & + & $\begin{array}{c}\text { Overall sunvival } \\
\text { PFS }\end{array}$ & $10.50 \%$ & Martens et al. 2016 \\
\hline & Ant-CTLA4 & 720 & $\uparrow$ Derived neutrophil-to-lymphocyte ratio (dNLR) & & Overall survival & 3 & Ferruccl et al. 2016 \\
\hline & AnD-CILAA & 58 & i Neutrophil-to-Iym phocye ratio (NLR) & $\cdot$ & PFS & 4 & Zaragoza et al.2016 \\
\hline & Anti-CTLA4 & 200 & $\downarrow$ Absolute monocyte count (AMC) & + & Overall sunvival & $650 / \mu \mathrm{l}$ & Martens et al. 2016 \\
\hline & Anti-CTLA4 & 134 & $\uparrow$ Number of organs involved & - & Overall sunvival & Continuous variable & Diem et al. 2015 \\
\hline & Anti-CTLA4 & 113 & $\uparrow 5100$ & - & Overall survival & $1 \times$ ULN & Kelderman etal. 2014 \\
\hline & Anti-CTLA4 & 120 & I C-reactive prote in during treatment & + & Response & $5 \mathrm{mg} / \mathrm{mL}$ & Simeone et al. 2014 \\
\hline & Ant-CTLA4 & 68 & I Myeloid derived supressor cells (MDSC) & + & Overall survival & Flow Cytom etry & Kitano etal.2014 \\
\hline & Anti-CT_A4 & 120 & I T-regulatorycells (Treg) & $\overline{-}$ & Response & $0.50 \%$ & Simeone et al. 2014 \\
\hline & & 200 & I T-regulatory cells (Treg) & + & Overall sunival & $1.50 \%$ & Martens et al. 2016 \\
\hline & Anti-CTLA4 & 144 & $\uparrow$ Circulating antibodies against NY-ESO-1 & + & Response & Titer $>100$ & Yuan et al. 2011 \\
\hline & & & † NY-ESO-1 specific CD8 T-cells & 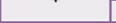 & Overall survival & Continuous veriable & \\
\hline
\end{tabular}

\section{TUMOR}

TCR

profiling
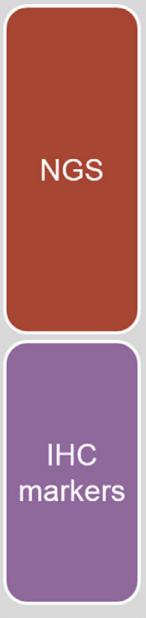

\begin{tabular}{|c|c|c|c|c|c|c|}
\hline Drug & \#patients & Markers & Correlation & Outcome & Method / Threshold & Reference \\
\hline Anti-PD1 & 46 & $\uparrow$ Clonal TCR repertoire & + & Response & NGS - CDR3 TCR $\beta-V J$ & Tumeh et al. 2014 \\
\hline \multirow{2}{*}{ Ant-PD1 } & 8 & $\uparrow$ TCR repertoire clonality under prior anti-CTLA4 & \multirow{2}{*}{+} & \multirow{2}{*}{ Response } & \multirow{2}{*}{ NGS - CDR3 TCRP-VJ } & \multirow{2}{*}{ Roh et al. 2017} \\
\hline & 56 & $\uparrow$ TCR repertoire clonallyat baseline & & & & \\
\hline Ant-PD1 & 10 & \TCR repertoire diversityindex & + & Response & NGS - CDR3 TCR $\beta$-VDJ & Inoue et al. 2016 \\
\hline Anti-PD1 & 34 & I TCR evenness in Ipl-naive, $\uparrow$ TCR richness in Ipi-progressive & + & Response & NGS-CDR3 TCRß-VJ & Riazetal. 2017 \\
\hline \multirow{3}{*}{ Anti-PD1 } & 68 pre- 41 & i Mutational load in Ipi-N & \multirow{3}{*}{+} & Overall sunival & \multirow{2}{*}{ WES } & \multirow{3}{*}{ Riazet al 2017} \\
\hline & therapy & I Mutational load and neoantigen load during therapy & & Response & & \\
\hline & 45 & Immune signature (T-cell activation and Iymphocye aggregation) & & Response & RNAseq & \\
\hline Anti-PD1 & 65 & I Mutational load and LRP1B mutation & + & Overall sunival & Targeted Panel Seq & Johnson etal. 2016 \\
\hline \multirow{2}{*}{ Anti-PD1 } & 38 & \multirow{2}{*}{ BRCA2 mutation, IPRES gene signature } & \multirow{2}{*}{+} & \multirow{2}{*}{ Response } & WES & \multirow{2}{*}{ Hugo et al. 2016} \\
\hline & 28 & & & & RNASEq & \\
\hline Antt-PD1 & 30 & $\uparrow$ Burden of copy number loss & - & Response & WES & Roh et al. 2017 \\
\hline Anti-CTLA4 & 174 & SERPINB3 or SERPINB4 mutation & + & Overall sunival & WES & Riazetal 2016 \\
\hline AntI-CTLAA & 64 & i Mutational load & + & Overall sunival & WES & Snyder et al. 2014 \\
\hline \multirow{2}{*}{ Ant-CTLAA } & 110 & \multirow{2}{*}{ † Mutational load, neoantigen load, cytolydic gene expression } & \multirow[t]{2}{*}{ + } & \multirow{2}{*}{ Overall sunival } & WES & \multirow{2}{*}{ Van Allen et al. 2015} \\
\hline & 40 & & & & RNAseq & \\
\hline \multirow{2}{*}{ Ant-CTLA4 } & 110 & \multirow{2}{*}{$\uparrow$ Aneuploidy } & \multirow{2}{*}{. } & $\begin{array}{l}\text { Response } \\
\text { Overall sunival }\end{array}$ & \multirow{2}{*}{ WES } & \multirow{2}{*}{ Davoli et al. 2017} \\
\hline & 64 & & & Overall sunival & & \\
\hline Ant-PD1 & 24 & 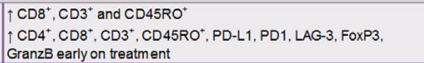 & + & Response & Immunohistochemis try & Chen etal. 2016 \\
\hline Ant-PD1 & 41 & १ PD-L1 expression & + & Response & Immunohistochemisty $(\geq 5 \%)$ & Taube et al. 2014 \\
\hline \multirow{2}{*}{ Ant-PD1 } & 210 & \multirow{2}{*}{$\uparrow P D-L 1$ expression } & \multirow{2}{*}{ + } & \multirow{2}{*}{$\begin{array}{c}\text { Overall survival } \\
\text { and PFS }\end{array}$} & Immunohis tochem isty ( $25 \%)$ & Robert et al. 2014 \\
\hline & 549 & & & & Immunohistochem istry ( $\geq 1 \%)$ & Robert tetal. 2015 \\
\hline Ant-PD1 & 35 & I Indoleam ine 2,3-dioxygenase (IDO) & + & Response & Immunohistochemis try & Hamid et al. 2011 \\
\hline Ant-PD1 & 46 & 1 T-cell infiltration in the tum or & + & Response & Immunohistochemis try & Tumeh etal. 2014 \\
\hline Ant-CTLA4 & 24 & $\uparrow \mathrm{CD} 8+\mathrm{T}$-cells early during treatment & + & Response & Immunohistochemistry & Chen etal. 2016 \\
\hline Anti-CTLA4 & 27 & $T$ T-cell infiltration in the tum or at 4 weeks & + & Response & Immunohistochemis try & Hamid et al. 2011 \\
\hline Ant-CTLA4 & 45 & $\begin{array}{l}\text { T Expression of immune related genes (CCL4, CCL5, CXCL., } \\
\text { CXCL10, CXCL11, IFN-y, IDO1, GBP1 MHC \| molecules, GBP1) }\end{array}$ & + & Response & $\begin{array}{l}\text { Affymetrix gene expression } \\
\text { analysis }\end{array}$ & Ji et al. 2012 \\
\hline
\end{tabular}

FIGURE 1 | Summary of the biomarker studies performed on peripheral blood and tumor on anti-CTLA4 and anti-PD1 treatment. 


\section{BIOMARKERS FROM NEXT-GENERATION SEQUENCING}

Whole exome (WES) and RNA sequencing (RNAseq) are powerful tools for evaluating the genomic landscape of a tumor. WES only captures the exonic gene regions, so it enriches for mutations in coding regions, while RNAseq can provide the entire transcriptome of a sample and is useful for establishing gene signatures for specific cohorts within a patient group. In terms of immunotherapy, WES has been useful in determining mutational load and discovering neoantigens in melanoma tumors (27). Melanoma has one of the highest mutation rates of all cancers (28-30) and has a high probability for neoantigen generation. Neoantigens are the result of somatic mutations which translate into a mutated protein that is detected and presented by APCs. Neoantigens are an attractive target for immunotherapy as they are only expressed by the tumor and not by the normal tissue. Many studies have utilized WES and RNAseq to evaluate the mutation profile and gene expression changes in patients treated with anti-CTLA-4 and anti-PD-1, with the aim to find biomarkers to predict response.

Snyder et al. performed WES on 64 patients treated with antiCTLA-4 (31). This study was the first to associate mutational burden to clinical benefit and they also defined a neoantigen signature associated with clinical benefit. They concluded that, although patients with high mutation burden are more likely to respond to anti-CTLA-4, the types of neoantigen the patient expresses will ultimately determine their response. Van Allen et al. performed WES on 110 patients and RNAseq on 40 patients treated with anti-CTLA-4 (32). They could confirm that mutational load is associated with clinical benefit to anti-CTLA-4 treatment. Neoantigen load was also measured and this parameter was also significantly associated with response; however, they could not detect the neoantigen signature seen in the study performed by Snyder and colleagues. They concluded that clinically beneficial neoantigens are most likely private events (specific to each individual) and recurrent neoantigens (consistent in the general population) are quite rare. Van Allen and colleagues also analyzed the transcriptome in a subset of these patients and found that expression of cytolytic markers, such as granzyme A and perforin, were beneficial for response. Expression of CTLA-4 and PD-L2 was also associated with clinical benefit. Riaz et al. performed WES on 174 patients treated with anti-CTLA-4 therapy (33). They discovered 48 patients with mutations in SERPINB3 or SERPIN4 and observed that those patients were more likely to be responders. Patients with SERPINB3 or SERPINB4 mutations also had higher mutational loads. Friedlander et al. performed a quantitative polymerase chain reaction (PCR) study on peripheral blood from 360 patients receiving anti-CTLA-4 therapy (34). From a panel of 169 genes, they established a 15 gene signature that was predictive and prognostic for response and 1-year OS to anti-CTLA- 4 treatment. The 15 genes are ITGA4, LARGE, CDK2, TIMP1, DPP4, NRAS, ERBB2, NAB2, ADAM17, RHOC, TGFB1, CDKN2A, HLADRA, MYC, and ICOS.

In order to elucidate resistance mechanisms and biomarkers of response to treatment, Hugo et al. used WES (38 patients) and RNAseq (28 patients) on a set of melanoma patients treated with anti-PD-1 (35). Mutational load did not have a significant association to response to anti-PD-1 therapy and neoantigen load was not significantly correlated with response either. Nonetheless, mutational load was associated with OS suggesting that other factors influence response to anti-PD-1 and survival. BRCA2 mutations occurred in $30 \%$ of the responders to anti-PD-1. RNAseq analysis uncovered a co-enrichment of 26 gene signatures in 9 of the 13 non-responding patients, which the authors termed innate anti-PD-1 resistance (IPRES) signature. They validated the IPRES signature on three other datasets and found over-representation in anti-PD-1 non-responding samples.

In a small study of four patients treated with anti-PD-1, Zaretsky et al. used WES on patients that developed new lesions under antiPD-1 therapy. They discovered that the progressive tumors acquired JAK1, JAK2, or B2M loss of function mutations. JAK1 and JAK2 mutations cause insensitivity to interferon gamma-induced arrest and B2M mutations led to a loss of MHC class 1 expression (36). In a follow-up study, Shin et al. performed WES on 23 patients before anti-PD-1 treatment (37). In their cohort, mutational load had no association to response and one of the non-responders had a loss of function mutation in JAK1. This study confirms the role of JAK1 as a marker for innate and adaptive resistance to anti-PD-1, although it might be a rare occurrence.

Roh et al. performed WES on sequential biopsies of patients treated with anti-CTLA- 4 and then anti-PD-1. Thirty patients had WES at baseline, 3 on anti-CTLA- 4 treatment, 25 at antiCTLA-4 progression, 18 on anti-PD- 1 treatment, and 12 at anti-PD-1 progression. Overall, they found that mutation burden was not associated with response, but high copy number loss was associated with poor response (38). In the regions with recurrent copy number loss, PTEN was one of the notable tumor suppressor genes suggesting that it could be a driver of resistance mechanisms to immunotherapy. Another study also observed that PTEN loss was associated with resistance to anti-CTLA-4 therapy (39).

Johnson et al. performed targeted panel sequencing on 65 patients treated with anti-PD-1 therapy. In their cohort, mutational load was associated with response to anti-PD-1 and patients with high mutational load (>23.1 mutations/MB) had longer PFS and OS compared to the intermediate mutational load group (3.3-23.1 mutations/MB) and the low mutation load $(<3.3$ mutations/MB) group. They observed more frequent BRCA2 mutations in responders than in non-responders $(5 / 32$ vs. 2/33). LRP1B mutations were significantly enriched in the responder group (11/32) compared to the non-responder group $(1 / 33)$. LRP1B mutated patients also had a higher mutational load compared to LRP1B wild-type patients.

Riaz et al. performed WES on 68 patients treated with antiPD- 1 that had previously progressed on anti-CTLA-4 therapy (35 patients) or were naïve to anti-CTLA-4 (33 patients). Mutational load was associated with clinical benefit in the anti-CTLA-4naive group, but not the anti-CTLA-4-resistant group. No single gene mutations were significantly associated with response or resistance to therapy. Decreased mutational and neoantigen load during therapy was associated with response in both anti-CTLA4-naïve and anti-CTLA-4-resistant groups. RNAseq analysis of the pretreatment samples showed an enrichment in T-cell activation and lymphocyte aggregation pathways. These signatures 
indicate an immunologically active tumor, or "hot tumor," and all patients with complete response or partial response in the anti-CTLA-4-resistant group had the "hot tumor" signature, although not all responders in the anti-CTLA-4-naïve group had the "hot tumor" signature. Riaz et al. also investigated the early effects of anti-PD-1 treatment (29 days after start) by RNAseq and uncovered a global increase in immune checkpoint genes such as PD-1, CTLA-4, CD274 (PD-L1), ICOS, and LAG3 in all samples. For responders, the significant pathways included inflammatory response and cytokine-mediate signaling pathways.

Finally, Davoli et al. investigated the role of aneuploidy in response to immunotherapy (40). They analyzed the copy number data from 5,255 tumor/normal samples, representing 12 cancer types from The Cancer Genome Atlas project, and found that for most tumors, there was a positive correlation between aneuploidy severity and mutational load. They also found that tumors with high levels of aneuploidy showed elevated expression of cell cycle and cell proliferation markers, as well as a reduced expression of markers for cytotoxic immune cell infiltrates. Aneuploidy levels were a stronger predictor of markers of cytotoxic immune cell infiltration than tumor mutational load. To correlate aneuploidy with response to immunotherapy, they used data from Snyder et al. and Van Allen et al. $(31,32)$ and found in both datasets that high levels of aneuploidy correlated with poorer survival.

\section{BIOMARKERS FROM T-CELL RECEPTOR (TCR) PROFILING}

Antigen detection by T-cells is by definition dependent on tumorspecific T-cell generation and clonal amplification. In the context of immunotherapies, which aim at enhancing the recognition of the cancer cell by the immune system, there is an obvious rational basis for examining the T-cell repertoire in order to shed light on the specific mode of action of the drug and find potential biomarkers of response.

The main challenge when analyzing TCR repertoire is its immense diversity. The TCR is a heterodimer comprised of two chains $\alpha \beta$ or $\delta \gamma$. The $\beta$ and $\delta$ chains, are generated by the random rearrangement of a variable region $(\mathrm{V})$, a diversity region (D), and a joining region $(\mathrm{J})$ with a constant region (C). The $\alpha$ and $\gamma$ chains, consists of segments from the $\mathrm{V}$, J, and $\mathrm{C}$ regions. Additional complexity is introduced by random addition or deletion of nucleotides at the junction sites of $\mathrm{V}, \mathrm{D}$, and J. The theoretical limit of the TCR repertoire is in the range of $10^{15}$, which is several magnitudes higher than the total amount of T-cells in the body, approximately $4 \times 10^{11}$ (41). The estimated number for the TCR repertoire is in the order of $10^{6}$ to $10^{8}$ (42). Most of the studies mainly assess the complementarity-determining region 3 (CDR3) from $\beta$ chain, which is considered an acceptable proxy for estimating diversity since it is the most variable region of the receptor and that $\alpha \beta$ T-cells represent about $90 \%$ of all T-cells (43). Due to the advances in NGS, it is possible now to identify each individual TCR sequence in the CDR3 region (44). Multiplex PCR is one of the widely used methods to amplify the CDR3 region. Primers for the J alleles or the constant region of the TCR $\alpha$ and $\beta$ chains are used together with a mix of primers for all known $\mathrm{V}$ alleles.
A drawback of multiplex PCR is that it is limited to known $V$ alleles. As a result, for TCR discovery experiments, other methods such as targeted enrichment-a technique where RNA baits capture the TCR receptor, usually the CDR3 region-are preferred. Since bait capture takes into account mismatches, it allows for discovery of new alleles and TCR receptors. In the context of immunotherapy, TCR repertoire analysis is useful for determining if the tumorreactive clones have undergone activation and clonal expansion. In an adequate immune response, the tumor-specific T-cells will represent a significant proportion of the whole repertoire and therefore be assessable at the level of the whole TCR population.

In 2014, Robert et al. compared pre- to post-treatment peripheral blood mononuclear cell (PBMC) samples from melanoma patients treated with anti-CTLA-4 (45). The results from deep sequencing of the multiplex PCR for the TCR V $\beta$ CDR3 region showed that 19 out of 21 patients had an increased number of unique clonotype (richness). There was no significant difference in the $\mathrm{V}$ or $\mathrm{J}$ segment usage and no difference in the total of unique sequences between responders and non-responders. The number of unique productive sequences in the top $25 \%$ of clones showed a particularly high increase in diversity after treatment. Those changes were not associated with peripheral lymphocyte count; however, $\mathrm{CD}^{+}$tumor-infiltrating cells showed a positive correlation with the TCR repertoire diversity. Finally, they showed that patients experiencing more toxicities had more diverse sequences post treatment. Overall, this study reports that anti-CTLA-4 treatment increases TCR repertoire diversity in an unspecific manner. Subsequently, the same group performed a similar study on 9 anti-PD-1-treated patients and compared the results (46). Unlike the effect of anti-CTLA-4, anti-PD-1 therapy does not increase TCR repertoire complexity, on the contrary, 4/9 samples show a decrease $>15 \%$ in the absolute number of unique sequences and only one had an increase $>15 \%$. Those results suggest that the mode of action of the two drugs is considerably different.

Cha et al. shed more light on the potential mechanism of anti-CTLA-4; their study assessed the changes in TCR repertoire between baseline and 4 weeks of treatment in PBMCs from 21 melanoma patients (47). They confirmed that anti-CTLA-4 treatment induces a significant change in the clonotypes frequency compared to healthy donors. They showed that the diversification is the result of a higher gain of new clonotypes and lower loss of existing ones. The number of therapy-induced expanding clones are not different between the responders and the non-responders, which is in line with what Robert et al. described. However, patients who survived longer exhibited less clonotypic changes overtime, they maintained the most abundant clones which were present at baseline and also had fewer clones significantly decreasing in frequency. Finally, they also demonstrated that the clones expanding in response to therapy are largely non-naïve T-cells, suggesting that patients who respond to the therapy already have pre-primed T-cells in circulation before the onset of anti-CTLA-4 treatment.

In light of these findings, Postow et al. hypothesized that the shape of the TCR repertoire prior to treatment initiation may influence the likelihood of a response to the treatment (48). Twelve baseline PBMC samples from 4 responders and 8 non-responders 
to anti-CTLA-4 were analyzed for richness and evenness of the TCR repertoire. In this small cohort, they first showed that patients who responded to the treatment have more similar VJ usage among each other than compared to the VJ usages in the non-responders. Furthermore, low richness or evenness of the TCR repertoire was significantly associated with a poor response to anti-CTLA-4. That is, a TCR repertoire composed of less unique clones (less diverse) or skewed toward a few specific clones (very clonal) is predictive of a non-response to the treatment.

The detection of clonally expanded tumor-specific T-cell in the blood indicates that the immune system mounted an immune response against a foreign entity, which could be the tumor. However, it is not certain that the activated T-cells would be able to home to the tumor efficiently and be able to kill the cancer cells. Therefore, it is also important to assess the immune status at the tumor site.

Tumeh et al. performed a very elegant study exploiting tumor samples from melanoma patients prior and during anti-PD-1 treatment (49). By qualitative and quantitative immunohistochemistry, they revealed that, at baseline, patients who eventually respond to the therapy, have more $\mathrm{CD}^{+} \mathrm{T}$-cells at the invasive margin of the tumor compared to non-responders. This population increases and migrates toward the center of the tumor during treatment in responders. They showed that an efficient response to anti-PD-1 therapy requires pre-existing $\mathrm{CD}^{+} \mathrm{T}$-cells, which are most likely tumor specific. To confirm this theory, they sequenced the TCR V $\beta$ region of tumor baseline samples and found that responders indeed had a more clonal TCR repertoire. On treatment, samples of responders showed significantly more clonal expansion than non-responders.

Johnson et al. used NGS to assess differences between baseline samples of responders and non-responders to anti-PD-1 therapy (50). They assessed mutational load as well as specific mutations differentially occurring in responders and non-responders. They also investigated the TCR repertoire clonality of 42 samples and did not find any association with response. However, it is important to mention that times of sample acquisition were not immediately before and after treatment. The timing was quite broad, the study allowed the inclusion of samples collected over 12 months before start of treatment and also after treatment initiation. When the analysis was performed only on samples obtained within 4 months of treatment initiation, the non-responder group was only represented by five samples, including one potential outlier. Nonetheless, they noticed a trend toward higher clonality at baseline in patients who eventually responded to the therapy.

Inoue et al. analyzed the TCR repertoire of 10 pre- and postanti-PD-1 tumor samples (51). They noticed that the clonotypes with a read frequency $>0.5 \%$ at baseline significantly increased after treatment in responders. The calculation of the diversity index highlighted a slight decrease in tumors of responders compared to non-responders, which suggests oligoclonal expansion of certain TCR clones.

More recently, Roh et al. published a complementary analysis on a cohort of patients for which they performed TCR sequencing (38). They analyzed tumor samples from melanoma patients treated sequentially with anti-CTLA-4 and anti-PD-1 via WES and TCR sequencing. The TCR clonality assay revealed that there was no significant difference between responders and non-responders, pre- or on-treatment with anti-CTLA-4. A subpopulation of the patients $(n=8)$ received anti-CTLA- 4 followed by anti-PD- 1 after progression. All three responders to anti-CTLA- 4 followed by antiPD-1 showed an increase in TCR clonality during anti-CTLA-4 treatment. In addition, higher TCR clonality was seen in the responders prior to treatment and on treatment with anti-PD-1.

Riaz et al. investigated the evolution of melanoma tumors and their microenvironment under anti-PD-1 therapy (52). Patients who had previously progressed on anti-CTLA-4 and were naïve to anti-CTLA-4 were included in the study. They performed TCR sequencing on 34 samples pre- and 4 weeks post- anti-PD-1 treatment. There were no statistically significant differences in the baseline samples of either group. On anti-PD-1 therapy, the anti-CTLA-4-pretreated group had increased TCR richness associated with response, whereas the anti-CTLA-4-naive group had decreased TCR evenness associated with response. In line with Roh et al., pretreatment with anti-CTLA-4 seems to increase the expansion of tumor-specific T-cell cells, which are additionally expanded during anti-PD-1 treatment.

\section{MASS CYTOMETRY (CyTOF)}

The advent of CyTOF has allowed a more comprehensible analysis of the whole immune system and will be an important asset for immune oncology (53). The basic principle of CyTOF is similar to conventional flow cytometry. The assay quantifies multiple protein expression markers at the single-cell level. In contrast to flow cytometry, the detection is not achieved by fluorophore excitation, but by stable mass isotope quantification. The transition isotope bound to the antibodies are analyzed by a time of flight mass spectrometer. CyTOF has some advantages over flow cytometry, namely, the high purity of the metal isotopes reduces background noise, eliminating spectral spillover and cellular autofluorescence associated with conventional flow cytometry. It also enables the detection of more markers in the same experiment, theoretically up to a hundred. Multiple samples can be analyzed at the same time thanks to a barcoding strategy (up to 20), and therefore reduce inter-sample variation. CyTOF has primarily been used to analyze peripheral blood from patients undergoing immunotherapy. A better characterization of the precise mode of action of those drugs is crucial to help overcoming and predicting resistance as well as contributing to optimal development of future combination therapies.

In 2015, Das et al. analyzed peripheral blood from melanoma patients undergoing immunotherapy with anti-CTLA-4, anti-PD-1, or the combination of the two (54). Samples were collected at baseline and after 3 weeks of treatment. In this early study, CyTOF was mainly used to further characterize the cell population of interest previously identified by flow cytometry. The analysis revealed that the $\mathrm{Ki}^{+} 7^{+}$cells, increasing after combination treatment, have a transitional memory T-cell-like phenotype. Additional experiments were performed using other techniques than CyTOF, which lead the authors to conclude that anti-PD-1 and anti-CTLA-4 have distinct effects on the immune system.

In the context of a clinical trial assessing the safety of combining radiotherapy and immunotherapy in melanoma patients, 
Hiniker et al. analyzed baseline and follow-up PBMC samples from 9 patients (3 progressive disease, 6 complete response/ partial response) (55). CyTOF analysis revealed that the level of $\mathrm{CD}^{+} \mathrm{T}$-cells expressing IL-2 were higher at baseline and in the follow-up samples of responding patients. The same was true for central memory $\mathrm{CD}^{+} \mathrm{T}$-cell levels. However, the cytokine production was not significantly different from the population seen in non-responding patients, thereby suggesting that the cells are not functionally different from the non-responders.

The first study to use CyTOF as a main technique for analyzing human melanoma patient samples was performed by WistubaHamprecht et al. in early 2017 (56). The analysis consisted in performing CyTOF on 28 PBMC samples from stage IV melanoma patients who received different courses of treatments. A higher frequency in the APC-like population had a positive association with OS, whereas a higher frequency in the MDSClike population showed negative association with OS. Overall, an equal abundance of MDSC- and APC-like cells is associated with better survival. The analysis of the T-cell compartment revealed that there was a clear interpatient heterogeneity in the $\mathrm{CD} 4^{+}$and $\mathrm{CD}^{+} \mathrm{T}$-cell compartments compared to the other compartments which have more homogenous frequencies between patients. Only one $\alpha \beta \mathrm{T}$-cell population had some prognostic potential: a higher level of early differentiated $\mathrm{CD} 4^{+} \mathrm{T}$-cell was correlated with poorer OS. In the natural killer cell compartment, a highly cytotoxic cell population tends to correlate with better OS. Finally, a comprehensive analysis of immune signatures of all the melanoma-associated phenotypes identified a specific cluster with high prognostic capacity, performing even better than LDH. This cluster is significantly associated with poor OS and represented by an overall lower diversity across all the compartments, and especially in the myeloid compartment.

Takeuchi et al. investigated the effect of immunotherapy in melanoma patients by comparing PBMCs from 4 different patients receiving anti-PD-1 (2 responders and 2 non-responders) (57). The panel was composed of 35 markers and they used highdimensional clustering to analyze the data. The main finding in this paper is that $\mathrm{CD} 4^{+}$and $\mathrm{CD} 4^{+} \mathrm{CD}^{+}$cell populations increase during therapy. $\mathrm{CD}^{+} \mathrm{CD} 27^{+} \mathrm{FAS}^{-}$central memory $\mathrm{T}$-cell were shown to expand in a higher proportion in responders than in non-responders. These results were validated in a separate cohort $(n=4)$.

More recently, Krieg et al. performed a comprehensive analysis, assessing the correlation between baseline peripheral immune signature and response to anti-PD-1 in melanoma patients (58). The cohort was composed of 20 patients from whom baseline and on treatment samples were obtained. They used an optimized immune marker panel and a customized, interactive bioinformatics pipeline in order to identify potential predictive biomarkers. Three different CyTOF panels were used: one for the phenotypic characterization of lymphocytes, one to assess the T-cell functions, and the third one to characterize monocytes, which consisted of 30, 26, and 25 markers, respectively. By performing hierarchical clustering of all the samples pooled together, they identified a differential marker expression in responders compared to non-responders. Further analysis, and validation in an independent, blinded cohort by conventional flow cytometry, revealed that, at baseline, responders had a higher frequency of classical monocytes and lower frequency of lymphocytes compared to the non-responders.

\section{OUTLOOK}

The development of high-throughput technologies such as NGS and CyTOF have allowed researchers and clinicians to evaluate hundreds to tens of thousands of genes from a bulk tumor to a single-cell level. NGS is an invaluable tool for analyzing mutations and copy number profiles, gene expression changes and gene signatures, epigenetic alterations, the TCR repertoire, and single-cell gene expression changes. The recent development of CyTOF has also allowed the analysis of many markers at a single-cell level. In the context of immunotherapy, these high dimensional datasets will enhance the discovery of novel biomarkers, prognostic markers, and resistance mechanisms.

Next-generation sequencing biomarker discovery for antiCTLA-4 treatment have uncovered that mutational load and neoantigen load are the most informative for response and OS, but they are not perfect biomarkers as some non-responders may also present with high mutational load. Aneuploidy could also help foresee response to anti-CTLA4 since it was highlighted as an independent predictor in a multivariate Cox model which included mutational load. Copy number analysis could as well be informative as loss in chromosome 10 was shown to be a poor prognostic marker in two studies. Many of these studies also analyzed tumor samples upon progression and found no recurrent genetic mutation, which could mean that resistance to anti-CTLA-4 is patient specific. In the context of anti-PD-1 treatment, mutational load is not a clear informative marker for response. As anti-PD-1 is a relatively new therapy, no large cohort studies with over 100 patients for NGS biomarker discovery have yet been performed. There are single patient examples showing that genes involved in the JAK-STAT pathway or antigen presentation could be predictive biomarkers for anti-PD-1 treatment. Loss of function mutations in JAK1, JAK2, and B2M are negative biomarkers for response and are involved in resistance to anti-PD-1 treatment in individual cases, but these mutations do not seem to be recurrent. RNAseq analysis from several studies suggest that tumors with high immune activity are more likely to respond to anti-PD-1. To better stratify patients for anti-CTLA- 4 or anti-PD- 1 treatment, a combinatorial approach investigating WES, copy number variation, and RNAseq would be needed.

Overall, most studies support that anti-CTLA-4 and antiPD-1 modulate TCR repertoire clonality upon treatment. This strengthens the notion that tumor-specific T-cell populations are affected by CTLA- 4 or PD-1 inhibition. In summary, most studies support that anti-CTLA-4 induces an expansion of clones in a non-specific manner and, therefore, broadens the TCR repertoire. On the other hand, anti-PD-1 seems to favor the proliferation of fewer specific clones giving rise to a more skewed repertoire, thereby suggesting that the baseline TCR repertoire of the patients plays a role in the response to the treatment. However, for the moment, those predictions arise mainly from early on-treatment evaluations that examined the evolution of the repertoire from baseline, as we are not yet able to precisely 
pinpoint the tumor-specific clones that, once clonally expanded, will facilitate tumor elimination. It is also important to highlight that the mode of action of the current immunotherapies are still debated and we do not fully comprehend their overall impact on different immune cell subpopulations. As a result, it is difficult to assess the global impact of the drugs on the immune response by investigating specific mechanisms individually. This is why high-throughput techniques discussed here are powerful emerging tools, which will allow us to elucidate this problem by looking at numerous markers simultaneously. The more we increase our knowledge of exact mechanisms, the better we will be able to exploit the therapies by using them in a targeted/ patient-specific manner. Interesting work by Twyman-Saint et al. combining anti-CTLA-4, anti-PD-1 and radiotherapy, underpins this assertion (59).

To our knowledge, despite the great potential held by CyTOF technology, to date, no research was published on the analysis of human melanoma tumor samples in the context of immunotherapies. One should however expect to see more forthcoming data, thanks to a novel exciting add-on technology that is starting to emerge. Indeed, a new laser system can be coupled to the CyTOF device which allows for imaging mass cytometry (60). That is, the detection of metal-labeled antibodies, as in standard CyTOF analysis, but performed on tissue sections by using multiplexed ion beam imaging. This state of the art technology will allow, not only to assess a high range of markers at the same time, but also to obtain spatial resolution and warrant a very comprehensive analysis of the cell-cell interaction in the tumor microenvironment. New developments of the system should soon facilitate

\section{REFERENCES}

1. Luke JJ, Flaherty KT, Ribas A, Long GV. Targeted agents and immunotherapies: optimizing outcomes in melanoma. Nat Rev Clin Oncol (2017) 14(8):463-82. doi:10.1038/nrclinonc.2017.43

2. Robert C, Long GV, Brady B, Dutriaux C, Maio M, Mortier L, et al. Nivolumab in previously untreated melanoma without BRAF mutation. $N$ Engl J Med (2015) 372(4):320-30. doi:10.1056/NEJMoa1412082

3. Maio M, Grob JJ, Aamdal S, Bondarenko I, Robert C, Thomas L, et al. Fiveyear survival rates for treatment-naive patients with advanced melanoma who received ipilimumab plus dacarbazine in a phase III trial. J Clin Oncol (2015) 33(10):1191-6. doi:10.1200/JCO.2014.56.6018

4. Hodi FS, O'Day SJ, McDermott DF, Weber RW, Sosman JA, Haanen JB, et al. Improved survival with ipilimumab in patients with metastatic melanoma. N Engl J Med (2010) 363(8):711-23. doi:10.1056/NEJMoa1003466

5. Robert C, Thomas L, Bondarenko I, O'Day S, Weber J, Garbe C, et al. Ipilimumab plus dacarbazine for previously untreated metastatic melanoma. N Engl J Med (2011) 364(26):2517-26. doi:10.1056/NEJMoa1104621

6. Wolchok JD, Chiarion-Sileni V, Gonzalez R, Rutkowski P, Grob JJ, Cowey CL, et al. Overall survival with combined nivolumab and ipilimumab in advanced melanoma. N Engl J Med (2017) 377:1345-56. doi:10.1056/NEJMoa1709684

7. Robert C, Schachter J, Long GV, Arance A, Grob JJ, Mortier L, et al. Pembrolizumab versus ipilimumab in advanced melanoma. $N$ Engl J Med (2015) 372(26):2521-32. doi:10.1056/NEJMoa1503093

8. Gardner D, Jeffery LE, Sansom DM. Understanding the CD28/CTLA-4 (CD152) pathway and its implications for costimulatory blockade. Am J Transplant (2014) 14(9):1985-91. doi:10.1111/ajt.12834

9. Dong H, Zhu G, Tamada K, Chen L. B7-H1, a third member of the B7 family, co-stimulates T-cell proliferation and interleukin-10 secretion. Nat Med (1999) 5(12):1365-9. doi:10.1038/70932

10. Latchman Y, Wood CR, Chernova T, Chaudhary D, Borde M, Chernova I, et al. PD-L2 is a second ligand for PD-1 and inhibits T cell activation. Nat Immunol (2001) 2(3):261-8. doi:10.1038/85330 the analysis of tumor samples in a similar fashion, while gaining spatial resolution to better interrogate the role of spatial interactions in immunotherapy response (with high throughput) (61).

In conclusion, the use of NGS and CyTOF has great potential to discover novel biomarkers for immunotherapy and the studies discussed above show exciting promises, but need to be further validated before clinical application. New prospective trials with large cohorts could include these technologies as a biomarker discovery platform and could validate many of these findings. In parallel, new algorithms to integrate multiple high dimensional datasets are being developed for a combinatorial biomarker approach, which could use these existing datasets as a training model. As NGS is becoming a standard service in many clinics, the development of next generation biomarkers should ultimately improve the stratification of patients for immunotherapy and thereby extend OS for these patients.

\section{AUTHOR CONTRIBUTIONS}

SH, ML, and PC conceptualized the manuscript and oversaw all aspects of its completing including writing, figure design, and literature review.

\section{FUNDING}

PC was supported by EU Horizon 2020 PHC grant No. 633974 (SOUND-Statistical multi-Omics UNDerstanding of Patient Samples). SH was supported a European Training Network MELGEN funded consortium No. 641458.

11. Ishida Y, Agata Y, Shibahara K, Honjo T. Induced expression of PD-1, a novel member of the immunoglobulin gene superfamily, upon programmed cell death. EMBO J (1992) 11(11):3887-95.

12. Michot JM, Bigenwald C, Champiat S, Collins M, Carbonnel F, Postel-Vinay S, et al. Immune-related adverse events with immune checkpoint blockade: a comprehensive review. Eur J Cancer (2016) 54:139-48. doi:10.1016/j.ejca.2015.11.016

13. Balch CM, Gershenwald JE, Soong SJ, Thompson JF, Atkins MB, Byrd DR, et al. Final version of 2009 AJCC melanoma staging and classification. J Clin Oncol (2009) 27(36):6199-206. doi:10.1200/JCO.2009.23.4799

14. Fantin VR, St-Pierre J, Leder P. Attenuation of LDH-A expression uncovers a link between glycolysis, mitochondrial physiology, and tumor maintenance. Cancer Cell (2006) 9(6):425-34. doi:10.1016/j.ccr.2006.04.023

15. Gershenwald JE, Scolyer RA, Hess KR, Sondak VK, Long GV, Ross MI, et al. Melanoma staging: evidence-based changes in the American Joint Committee on Cancer eighth edition cancer staging manual. CA Cancer J Clin (2017) 67(6):472-92. doi:10.3322/caac.21409

16. Kelderman S, Heemskerk B, van Tinteren H, van den Brom RR, Hospers GA, van den Eertwegh AJ, et al. Lactate dehydrogenase as a selection criterion for ipilimumab treatment in metastatic melanoma. Cancer Immunol Immunother (2014) 63(5):449-58. doi:10.1007/s00262-014-1528-9

17. Nosrati A, Tsai KK, Goldinger SM, Tumeh P, Grimes B, Loo K, et al. Evaluation of clinicopathological factors in $\mathrm{PD}-1$ response: derivation and validation of a prediction scale for response to PD-1 monotherapy. Br J Cancer (2017) 116(9):1141-7. doi:10.1038/bjc.2017.70

18. Jessurun CAC, Vos JAM, Limpens J, Luiten RM. Biomarkers for response of melanoma patients to immune checkpoint inhibitors: a systematic review. Front Oncol (2017) 7:233. doi:10.3389/fonc.2017.00233

19. Diem S, Kasenda B, Spain L, Martin-Liberal J, Marconcini R, Gore M, et al. Serum lactate dehydrogenase as an early marker for outcome in patients treated with anti-PD-1 therapy in metastatic melanoma. Br J Cancer (2016) 114(3):256-61. doi:10.1038/bjc.2015.467

20. Kaskel P, Berking C, Sander S, Volkenandt M, Peter RU, Krähn G. S-100 protein in peripheral blood: a marker for melanoma metastases: a prospective 
2-center study of 570 patients with melanoma. J Am Acad Dermatol (1999) 41(6):962-9. doi:10.1016/S0190-9622(99)70254-9

21. Diem S, Kasenda B, Martin-Liberal J, Lee A, Chauhan D, Gore M, et al. Prognostic score for patients with advanced melanoma treated with ipilimumab. Eur J Cancer (2015) 51(18):2785-91. doi:10.1016/j.ejca.2015.09.007

22. Simeone E, Gentilcore G, Giannarelli D, Grimaldi AM, Caraco C, Curvietto M, et al. Immunological and biological changes during ipilimumab treatment and their potential correlation with clinical response and survival in patients with advanced melanoma. Cancer Immunol Immunother (2014) 63(7):675-83. doi:10.1007/s00262-014-1545-8

23. Li Z, Feng J, Sun X. Is C-reactive protein a specific marker in melanoma? J Clin Oncol (2015) 33(31):3673-4. doi:10.1200/JCO.2015.62.2696

24. Nonomura Y, Otsuka A, Nakashima C, Seidel JA, Kitoh A, Dainichi T, et al. Peripheral blood Th9 cells are a possible pharmacodynamic biomarker of nivolumab treatment efficacy in metastatic melanoma patients. Oncoimmunology (2016) 5(12):e1248327. doi:10.1080/2162402X.2016.1248327

25. Yuan J, Hegde PS, Clynes R, Foukas PG, Harari A, Kleen TO, et al. Novel technologies and emerging biomarkers for personalized cancer immunotherapy. J Immunother Cancer (2016) 4:3. doi:10.1186/s40425-016-0107-3

26. Altman DG, McShane LM, Sauerbrei W, Taube SE. Reporting Recommendations for tumor marker prognostic studies (REMARK): explanation and elaboration. PLoS Med (2012) 9(5):e1001216. doi:10.1371/journal. pmed.1001216

27. Johnson DB, Sosman JA. Therapeutic advances and treatment options in metastatic melanoma. JAMA Oncol (2015) 1(3):380-6. doi:10.1001/jamaoncol. 2015.0565

28. Alexandrov LB, Nik-Zainal S, Wedge DC, Aparicio SA, Behjati S, Biankin AV, et al. Signatures of mutational processes in human cancer. Nature (2013) 500(7463):415-21. doi:10.1038/nature12477

29. Hayward NK, Wilmott JS, Waddell N, Johansson PA, Field MA, Nones K, et al. Whole-genome landscapes of major melanoma subtypes. Nature (2017) 545(7653):175-80. doi:10.1038/nature22071

30. Cancer Genome Atlas Network. Genomic classification of cutaneous melanoma. Cell (2015) 161(7):1681-96. doi:10.1016/j.cell.2015.05.044

31. Snyder A, Makarov V, Merghoub T, Yuan J, Zaretsky JM, Desrichard A, et al. Genetic basis for clinical response to CTLA-4 blockade in melanoma. N Engl J Med (2014) 371(23):2189-99. doi:10.1056/NEJMoa1406498

32. Van Allen EM, Miao D, Schilling B, Shukla SA, Blank C, Zimmer L, et al. Genomic correlates of response to CTLA-4 blockade in metastatic melanoma. Science (2015) 350(6257):207-11. doi:10.1126/science.aad0095

33. Riaz N, Havel JJ, Kendall SM, Makarov V, Walsh LA, Desrichard A, et al. Recurrent SERPINB3 and SERPINB4 mutations in patients who respond to anti-CTLA4 immunotherapy. Nat Genet (2016) 48(11):1327-9. doi:10.1038/ ng. 3677

34. Friedlander P, Wassmann K, Christenfeld AM, Fisher D, Kyi C, Kirkwood JM, et al. Whole-blood RNA transcript-based models can predict clinical response in two large independent clinical studies of patients with advanced melanoma treated with the checkpoint inhibitor, tremelimumab. J Immunother Cancer (2017) 5(1):67. doi:10.1186/s40425-017-0272-z

35. Hugo W, Zaretsky JM, Sun L, Song C, Moreno BH, Hu-Lieskovan S, et al. Genomic and transcriptomic features of response to anti-PD-1 therapy in metastatic melanoma. Cell (2016) 165(1):35-44. doi:10.1016/j.cell.2016.02.065

36. Zaretsky JM, Garcia-Diaz A, Shin DS, Escuin-Ordinas H, Hugo W, Hu-Lieskovan S, et al. Mutations associated with acquired resistance to PD-1 blockade in melanoma. N Engl J Med (2016) 375(9):819-29. doi:10.1056/ NEJMoa1604958

37. Shin DS, Zaretsky JM, Escuin-Ordinas H, Garcia-Diaz A, Hu-Lieskovan S, Kalbasi A, et al. Primary resistance to PD-1 blockade mediated by JAK1/2 mutations. Cancer Discov (2017) 7(2):188-201. doi:10.1158/2159-8290.CD$16-1223$

38. Roh W, Chen PL, Reuben A, Spencer CN, Prieto PA, Miller JP, et al. Integrated molecular analysis of tumor biopsies on sequential CTLA-4 and PD-1 blockade reveals markers of response and resistance. Sci Transl Med (2017) 9(379). doi:10.1126/scitranslmed.aah3560

39. Mohammad KS, Javelaud D, Fournier PG, Niewolna M, McKenna CR, Peng XH, et al. TGF-beta-RI kinase inhibitor SD-208 reduces the development and progression of melanoma bone metastases. Cancer Res (2011) 71(1):175-84. doi:10.1158/0008-5472.CAN-10-2651
40. Davoli T, Uno H, Wooten EC, Elledge SJ. Tumor aneuploidy correlates with markers of immune evasion and with reduced response to immunotherapy. Science (2017) 355(6322):eaaf8399. doi:10.1126/science.aaf8399

41. Jenkins MK, Chu HH, McLachlan JB, Moon JJ. On the composition of the preimmune repertoire of $\mathrm{T}$ cells specific for peptide-major histocompatibility complex ligands. Annu Rev Immunol (2010) 28:275-94. doi:10.1146/ annurev-immunol-030409-101253

42. Qi Q, Liu Y, Cheng Y, Glanville J, Zhang D, Lee JY, et al. Diversity and clonal selection in the human T-cell repertoire. Proc Natl Acad Sci U S A (2014) 111(36):13139-44. doi:10.1073/pnas.1409155111

43. Laydon DJ, Bangham CR, Asquith B. Estimating T-cell repertoire diversity: limitations of classical estimators and a new approach. Philos Trans $R$ Soc Lond B Biol Sci (2015) 370(1675):20140291. doi:10.1098/rstb.2014.0291

44. Rosati E, Dowds CM, Liaskou E, Henriksen EKK, Karlsen TH, Franke A. Overview of methodologies for T-cell receptor repertoire analysis. BMC Biotechnol (2017) 17(1):61. doi:10.1186/s12896-017-0379-9

45. Robert L, Tsoi J, Wang X, Emerson R, Homet B, Chodon T, et al. CTLA4 blockade broadens the peripheral T-cell receptor repertoire. Clin Cancer Res (2014) 20(9):2424-32. doi:10.1158/1078-0432.CCR-13-2648

46. Robert L, Harview C, Emerson R, Wang X, Mok S, Homet B, et al. Distinct immunological mechanisms of CTLA-4 and PD-1 blockade revealed by analyzing TCR usage in blood lymphocytes. Oncoimmunology (2014) 3:e29244. doi:10.4161/onci.29244

47. Cha E, Klinger M, Hou Y, Cummings C, Ribas A, Faham M, et al. Improved survival with $\mathrm{T}$ cell clonotype stability after anti-CTLA-4 treatment in cancer patients. Sci Transl Med (2014) 6(238):238ra70. doi:10.1126/scitranslmed. 3008211

48. Postow MA, Manuel M, Wong P, Yuan J, Dong Z, Liu C, et al. Peripheral T cell receptor diversity is associated with clinical outcomes following ipilimumab treatment in metastatic melanoma. JImmunother Cancer (2015) 3:23. doi:10.1186/s40425-015-0070-4

49. Tumeh PC, Harview CL, Yearley JH, Shintaku IP, Taylor EJ, Robert L, et al. PD-1 blockade induces responses by inhibiting adaptive immune resistance. Nature (2014) 515(7528):568-71. doi:10.1038/nature13954

50. Johnson DB, Frampton GM, Rioth MJ, Yusko E, Xu Y, Guo X, et al. Targeted next generation sequencing identifies markers of response to PD-1 blockade. Cancer Immunol Res (2016) 4(11):959-67. doi:10.1158/2326-6066. CIR-16-0143

51. Inoue $\mathrm{H}$, Park JH, Kiyotani $\mathrm{K}$, Zewde M, Miyashita A, Jinnin M, et al. Intratumoral expression levels of PD-L1, GZMA, and HLA-A along with oligoclonal $\mathrm{T}$ cell expansion associate with response to nivolumab in metastatic melanoma. Oncoimmunology (2016) 5(9):e1204507. doi:10.1080/2162 402X.2016.1204507

52. Riaz N, Havel JJ, Makarov V, Desrichard A, Urba WJ, Sims JS, et al. Tumor and microenvironment evolution during immunotherapy with nivolumab. Cell (2017) 171(4):934-949.e15. doi:10.1016/j.cell.2017.09.028

53. Chang S, Kohrt H, Maecker HT. Monitoring the immune competence of cancer patients to predict outcome. Cancer Immunol Immunother (2014) 63(7):713-9. doi:10.1007/s00262-014-1521-3

54. Das R, Verma R, Sznol M, Boddupalli CS, Gettinger SN, Kluger H, et al. Combination therapy with anti-CTLA-4 and anti-PD-1 leads to distinct immunologic changes in vivo. J Immunol (2015) 194(3):950-9. doi:10.4049/jimmunol. 1401686

55. Hiniker SM, Reddy SA, Maecker HT, Subrahmanyam PB, Rosenberg-Hasson Y, Swetter SM, et al. A prospective clinical trial combining radiation therapy with systemic immunotherapy in metastatic melanoma. Int J Radiat Oncol Biol Phys (2016) 96(3):578-88. doi:10.1016/j.jirobp.2016.07.005

56. Wistuba-Hamprecht K, Martens A, Weide B, Teng KW, Zelba H, Guffart E, et al. Establishing high dimensional immune signatures from peripheral blood via mass cytometry in a discovery cohort of stage IV melanoma patients. J Immunol (2017) 198(2):927-36. doi:10.4049/jimmunol.1600875

57. Takeuchi Y, Tanemura A, Tada Y, Katayama I, Kumanogoh A, Nishikawa H. Clinical response to PD-1 blockade correlates with a sub-fraction of peripheral central memory CD4+ T cells in patients with malignant melanoma. Int Immunol (2018) 30(1):13-22. doi:10.1093/intimm/dxx073

58. Krieg C, Nowicka M, Guglietta S, Schindler S, Hartmann FJ, Weber LM, et al. High-dimensional single-cell analysis predicts response to anti-PD-1 immunotherapy. Nat Med (2018) 24(2):144-53. doi:10.1038/nm.4466 
59. Twyman-Saint Victor C, Rech AJ, Maity A, Rengan R, Pauken KE, Stelekati E, et al. Radiation and dual checkpoint blockade activate non-redundant immune mechanisms in cancer. Nature (2015) 520(7547):373-7. doi:10.1038/ nature14292

60. Schapiro D, Jackson HW, Raghuraman S, Fischer JR, Zanotelli VRT, Schulz D, et al. histoCAT: analysis of cell phenotypes and interactions in multiplex image cytometry data. Nat Methods (2017) 14(9):873-6. doi:10.1038/ nmeth.4391

61. Di Palma S, Bodenmiller B. Unraveling cell populations in tumors by single-cell mass cytometry. Curr Opin Biotechnol (2015) 31:122-9. doi:10.1016/j.copbio. 2014.07.004
Conflict of Interest Statement: The authors declare that the research was conducted in the absence of any commercial or financial relationships that could be construed as a potential conflict of interest.

Copyright $\odot 2018$ Hogan, Levesque and Cheng. This is an open-access article distributed under the terms of the Creative Commons Attribution License (CC BY). The use, distribution or reproduction in other forums is permitted, provided the original author(s) and the copyright owner are credited and that the original publication in this journal is cited, in accordance with accepted academic practice. No use, distribution or reproduction is permitted which does not comply with these terms. 